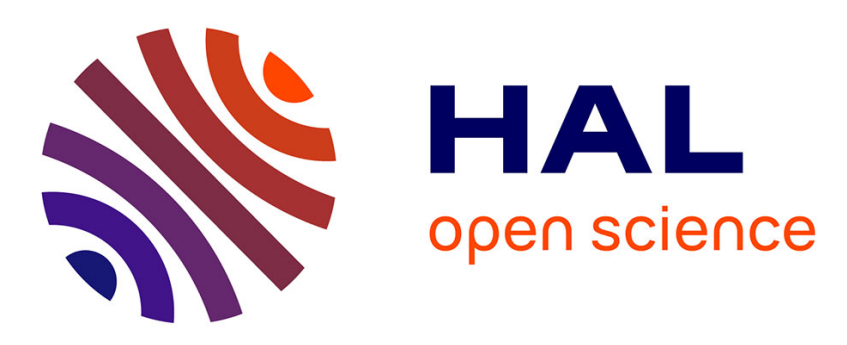

\title{
On-chip characterization of the viscoelasticity of complex fluids using microcantilevers
}

Mohamed Youssry, Etienne Lemaire, Benjamin Caillard, Annie Colin, Isabelle Dufour

\section{- To cite this version: \\ Mohamed Youssry, Etienne Lemaire, Benjamin Caillard, Annie Colin, Isabelle Dufour. On-chip char- acterization of the viscoelasticity of complex fluids using microcantilevers. Measurement Science and Technology, 2012, 23, 125306 (10 p.). 10.1088/0957-0233/23/12/125306 . hal-00745004}

\section{HAL Id: hal-00745004 https://hal.science/hal-00745004}

Submitted on 13 Nov 2014

HAL is a multi-disciplinary open access archive for the deposit and dissemination of scientific research documents, whether they are published or not. The documents may come from teaching and research institutions in France or abroad, or from public or private research centers.
L'archive ouverte pluridisciplinaire HAL, est destinée au dépôt et à la diffusion de documents scientifiques de niveau recherche, publiés ou non, émanant des établissements d'enseignement et de recherche français ou étrangers, des laboratoires publics ou privés. 
Preprint: Measurement Science and Technology, Vol. 23, 2012, 125306 (10pp)

\title{
On-chip characterization of the viscoelasticity of complex fluids using microcantilevers
}

Mohamed Youssry, ${ }^{1}$ Etienne Lemaire, ${ }^{2}$ Benjamin Caillard, ${ }^{2}$ Annie Colin, ${ }^{1}$ Isabelle Dufour*2

\author{
${ }^{1}$ Université de Bordeaux, LOF-Rhodia, UMR 5258, F-33600 Pessac, France \\ ${ }^{2}$ Université de Bordeaux, IMS, UMR 5218, F-33400 Talence, France
}

\begin{abstract}
Due to the need for a microrheometer monitoring the high-frequency viscoelasticity of soft matter in situ, we describe a cantilever-based microrheometer to achieve this purpose. The elastic and viscous moduli of complex fluids can be measured with an acceptable accuracy over a high frequency bandwidth of 1-100 kHz. Some preliminary data on small samples $(\sim 10-100 \mu \mathrm{L})$ of simple Newtonian and viscoelastic polymer and surfactant solutions showed the ability to measure the dynamic moduli in the range of 0.01 $10 \mathrm{kPa}$. This approach will provide a new way to characterize in situ, dynamic microrheology of minute and trace materials and will advance the field of biorheology, microfluidics, and polymer processing.
\end{abstract}

\section{* Corresponding author}

\section{Prof. Isabelle Dufour}

Université de Bordeaux, IMS, UMR 5218, F-33400 Talence, France.

Tel. +33540006554, Fax. +33556371545

E-mail : isabelle.dufour@ims-bordeaux.fr 
Preprint: Measurement Science and Technology, Vol. 23, 2012, 125306 (10pp)

\section{INTRODUCTION}

Rheology is the study of deformation and flow of matter. True to its name, microrheology simply refers to "rheology on the micrometer (length), microliter (volume) or microsecond (time) scales". The continuing need to quantify material properties at the micro-scale drives researchers to find a technique that satisfies this objective, since the traditional mechanical rheometers are limited to a small low-frequency range (typically $<100 \mathrm{~Hz}$ ) and require relatively large samples (tens of milliliters). In the last decade, opticsbased microrheology techniques, such as quasielastic light scattering (QELS), diffusing wave spectroscopy (DWS), and video and laser particle tracking techniques, have proved to be powerful in studying the high-frequency viscoelastic properties of various complex fluids. These techniques are classified as passive microrheology, i.e., ones in which the linear viscoelastic regime is attained, since they are based on the application of the fluctuation-dissipation theorem in which the mean-square displacement (MSD) of a thermally-induced probe particle is monitored and directly related to the viscoelasticity of the materials (at thermal equilibrium) through a generalized Stokes-Einstein relation (GSER) [1,2]. The continuing developments in the theory and instrumentation of these techniques have resulted in the elimination of errors and limitations that had been introduced in the infancy of these techniques. However, some important issues still remain concerning probe particle-fluid interactions and their influence on the viscoelasticity of the studied liquid, their complicated setup and rearrangements, and lengthy calibration and data treatment.

Microcantilevers fall under the category of MicroElectroMechanical Systems (MEMS) and rely primarily on mechanical phenomena and involve the transduction of the mechanical energy for detection [3]. They have received a great deal of attention for use as chemical sensors in gas or liquid media and as biosensors [4-10], viscometers [11-15] and dynamic rheometers [16]. Since Atomic Force Microscopy (AFM) has been used to study the dynamics of biological materials qualitatively [17], considerable attempts have been devoted to AFM as a probe to study the viscoelasticity of complex fluids quantitatively [1822]. However, these attempts are only restricted to the low frequency range. With AFM, the attachment of a colloidal sphere to the cantilever of the microscope provides highly 
accurate measurement of the hydrodynamic interaction between the probe particle and the fluid [18]. These rheological functions can be subsequently modeled with standard rheological theory and compared with bulk measurements. Based on this approach, Belmiloud and coauthors proposed an analytical model based on a simplified hydrodynamic function [23] to estimate the material properties (viscosity and density) using the vibrating phenomena of silicon-based microcantilevers [14,24]. (This principle is extended in the current study by taking into account the elastic component of the fluid, as detailed Sec. III) The microcantilever successfully proved to operate as a microviscometer probe with an acceptable accuracy for measuring the viscosity of various liquids $[11,12,24]$. In the present study, we extend the theoretical model [14] to exploit the vibrating phenomena of microcantilevers in an attempt to probe the linear viscoelastic spectra of complex fluids, i.e., to test the feasibility of microcantilevers operating as microrheometers.

The rheology of complex fluids is crucial for both applications and basic science since flow-structure relationships are of significant interest as they can provide a key understanding of the stability and performance of various formulations. Among those complex fluids of particular interest are synthetic and biopolymer dispersions and surfactant giant and wormlike micellar (WLM) solutions. These deserve significant attention due to their rich dynamics and unique characteristic mesoscopic structures, which make them ideal complex fluids to study from both fundamental and applications viewpoints. In this context, we test our novel method on various polymer and surfactant WLM solutions, trying to examine its validity in probing the high-frequency viscoelasticity and comparing/combining these results with those of low-frequency probes obtained by dynamic macrorheometry to understand both the slow and fast dynamics-microstructure relationships. The motivation for this study arises from the recent interest in developing microrheological techniques able to overcome the drawbacks introduced by the previously mentioned techniques. Moreover, arrays of several cantilevers covering a broad frequency bandwidth can be integrated on a chip to interrogate microliter-scale volumes of expensive and rare biological fluids for in situ microrheological measurements, thus providing another significant advantage of pursuing a microcantilever-based microrheometer. 
Preprint: Measurement Science and Technology, Vol. 23, 2012, 125306 (10pp)

\section{MATERIALS AND METHODS}

Cetylpyridinium chloride monohydrate $(\mathrm{CPyCl})$ and sodium salicylate (NaSal; 99.5\%) were purchased from VWR, Belgium, cetyltrimethylammonium bromide (CTAB; $>99 \%$ ) from G-Biosciences, USA, sodium chloride ( $\mathrm{NaCl} ; \geq 99 \%)$ from Acros Organics, and polyethylene oxide (PEO; $400 \mathrm{kDa}$ ) and silicone oil from Sigma-Aldrich. All materials have been used without further purification. The surfactant solutions were prepared by mixing the appropriate weights of components (Table 1) with deionized water under continuous stirring for two days, after which the samples were stored for one week at room temperature to relax sufficiently prior to performing the experiments. The polymer solution was prepared by mixing the PEO powder with deionized water and stirring the mixture for two weeks at $40{ }^{\circ} \mathrm{C}$. A drop of chloroform was added to prevent bacterial growth, and the solution was left at rest for two weeks prior to any measurements.

Table 1. The composition of surfactant solutions used in this study.

\begin{tabular}{|l|c|c|c|}
\hline Sample & {$[$ Surfactant $](\mathrm{mM})$} & {$[$ NaSal $](\mathrm{mM})$} & {$[\mathrm{NaCl}](\mathrm{mM})$} \\
\hline CPyCl1 & 28 & 14 & 500 \\
\hline CPyCl2 & 112 & 56 & 0.5 \\
\hline CTAB1 & 100 & 40 & - \\
\hline
\end{tabular}

Table 2. Dimensions ( $L=$ length, $b=$ width, $h=$ thickness) and resonant frequency in air for the microcantilevers used.

\begin{tabular}{|c|c|c|c|c|}
\hline Cantilever & $L(\mu \mathrm{m})$ & $b(\mu \mathrm{m})$ & $h(\mu \mathrm{m})$ & $f_{r}(\mathrm{kHz})$ \\
\hline LL & 2810 & 100 & 20 & 3.5 \\
\hline LH & 1440 & 285 & 20 & 12 \\
\hline A & 500 & 100 & 20 & 92 \\
\hline
\end{tabular}

The low-frequency viscoelastic behavior (macrorheology) of the examined complex fluids has been investigated using a stress-controlled rheometer AR-G2 (TA Instruments) equipped with a cone and plate geometry (plate diameter $40 \mathrm{~mm}$, cone angle $0.03 \mathrm{rad}$, gap size $59 \mu \mathrm{m})$. The temperature was controlled by Peltier system $\left( \pm 0.1^{\circ} \mathrm{C}\right)$. All measurements have been done at a fixed temperature of $20^{\circ} \mathrm{C}$, at which no change in the homogeneity of 
the mixtures was observed, thus indicating that the surfactant/cosurfactant mixtures were far above their respective Krafft temperatures.

The high-frequency viscoelasticity (microrheology) of the complex fluids has been studied based on an experimental setup as follow. Chips with rectangular silicon-based microcantilevers were provided by LAAS-CNRS at Toulouse, France. A series of microcantilevers were used for measurements, namely LL, LH and A, having the different geometries specified in Table 2 (due to the use of SOI wafers the silicon thicknesses of all microcantilevers are the same). The microcantilevers have been designed in order to be actuated electromagnetically by the Lorentz force. For this purpose, a conducting path has been deposited on the top surface of the microcantilever (figure 1). The conducting path is made of a titanium layer of $100 \mathrm{~nm}$ thickness covered by a gold layer of $700 \mathrm{~nm}$ thickness. The width of the conducting path is $10 \mu \mathrm{m}$ and is placed at $10 \mu \mathrm{m}$ from the edge of the cantilever. The electromagnetic actuation is obtained by the use of either one (or two) magnet(s) generating a continuous magnetic field and an alternative voltage generating an alternative electrical current in the conducting path. Then, the Lorentz force causes the microcantilever deflection at the same frequency as the actuation voltage. In order to be able to make measurement in electrically conductive fluids, a layer of silicon oxide is put on the top surface of the cantilever (the one with the conducting path for electromagnetic actuation): the thickness of this insulating layer is $600 \mathrm{~nm}$.

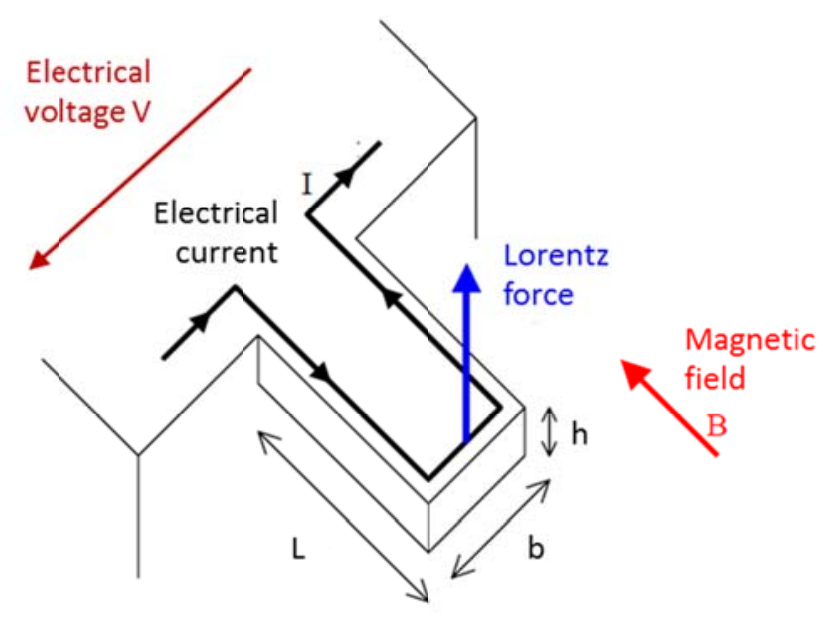

Figure 1. Schematic representation of the microcantilever with a conducting path allowing the electromagnetic actuation using a magnet and an alternative voltage. 
The silicon chip containing the cantilever was fixed on a Teflon reservoir in which the microcantilever was completely immersed in the fluid under test (figure 2). A few hundred microliters of fluid were sufficient to completely immerse the cantilever. At the bottom of the reservoir two permanent magnets were fixed to induce an electromagnetic actuation upon application of a small voltage to the cantilever conducting path. The cell was then connected to a Scanning Laser Doppler Vibrometer (Micro-System Analyzer MSA-500 from Polytec).

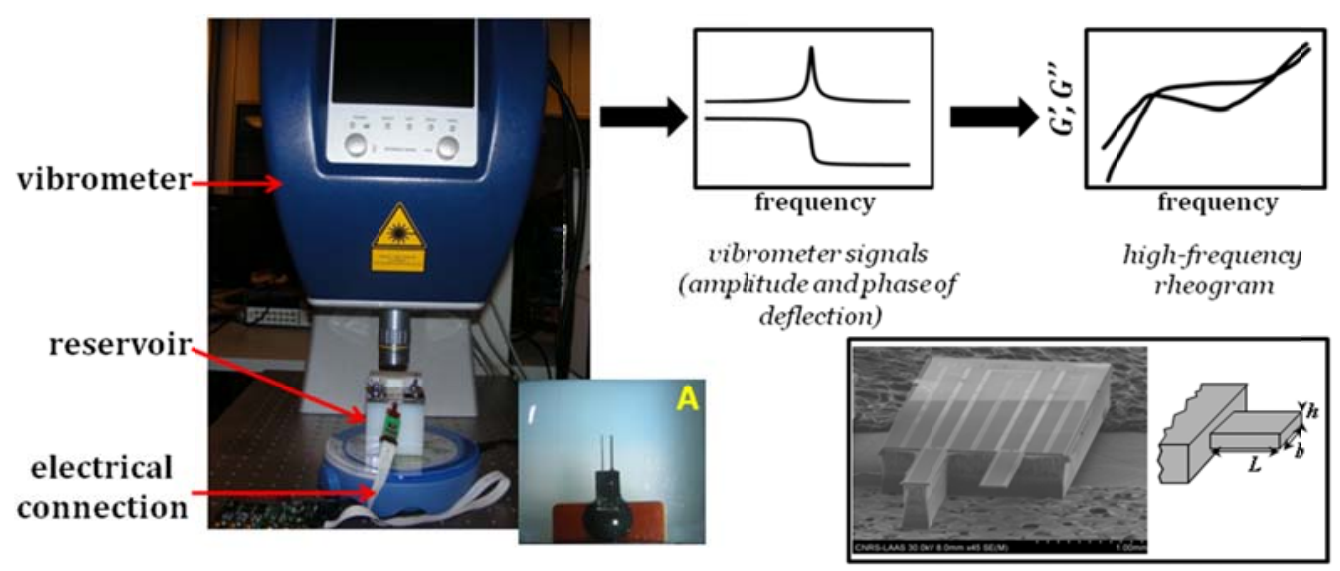

Figure 2. Schematic representation explaining the experimental setup (left), the data treatment (top right) and the cantilever SEM view (right bottom). The photo inset A is a top view of the reservoir showing the cantilever immersed in fluid.

The vibrometer is a powerful tool for quickly characterizing out-of-plane and inplane dynamic behavior of microdevices up to several $\mathrm{MHz}$ without contact. Laser Doppler vibrometry allows non-contact measurement in real time for the characterization of out-ofplane vibrational behavior and for determining the vibration velocity and displacement at any sample point. With the Polytec MSA500 the displacement resolution is in the range of picometer. For measurements in liquid media in the present study, the signals were collected by averaging the results of 100 runs during $c a$. 5-10 min. All measurements were done in a clean room at fixed temperature $\left(19.7 \pm 0.5^{\circ} \mathrm{C}\right)$ and humidity $(40 \%)$ levels.

Figure 2 summarizes the overall approach of the new procedure for studying the viscoelasticity of complex fluids over a high frequency bandwidth. For a cantilever immersed in a fluid, the amplitude and phase of deflection spectra are treated analytically 
to extract the characteristic viscoelastic properties of the fluid (the elastic modulus $G^{\prime}$, viscous modulus $G^{\prime \prime}$, and the complex viscosity $\eta^{*}$ ) over an extended frequency range, typically from 1 to $100 \mathrm{kHz}$ depending on the cantilever geometry. Each cantilever provides a deflection spectrum over a specified frequency range which can be significantly extended by merging the spectra of different cantilevers to cover broader range.

\section{THEORY}

For a vibrating cantilever actuated by an external driving force and immersed in an incompressible fluid, the drag force per unit length exerted on the surfaces of the cantilever beam is a sum of inertial and viscous forces proportional, respectively, to the acceleration and velocity of the beam, and can be written, in the frequency domain, as follows [25]:

$$
F_{\text {fluid }}=-j 2 \pi f g_{1}(f) w+4 \pi^{2} f^{2} g_{2}(f) w
$$

where $w$ is the amplitude of deflection at the considered location along the microcantilever and $f$ is the excitation frequency. The terms $g_{1}$ and $g_{2}$ correspond, respectively to the viscous and inertial forces and are related to the imaginary and real parts of the hydrodynamic function [26]:

$$
\begin{gathered}
g_{1}=\frac{\pi^{2} \rho_{f} b^{2} f}{2} \Gamma_{i}(f) \\
g_{2}=\frac{\pi \rho_{f} b^{2}}{4} \Gamma_{r}(f)
\end{gathered}
$$

where $\Gamma_{\mathrm{i}}$ and $\Gamma_{\mathrm{r}}$ are the imaginary and real parts of the hydrodynamic function, $\rho_{f}$ is the fluid mass density and $b$ is the cantilever width. For a uniform rectangular cantilever with a finite thickness, Maali et al. [23] simplified the hydrodynamic function of a ribbon geometry proposed by Sader [27] to be:

$$
\Gamma_{r}=a_{1}+a_{2} \frac{\delta}{b}
$$


Preprint: Measurement Science and Technology, Vol. 23, 2012, 125306 (10pp)

$$
\Gamma_{i}=b_{1} \frac{\delta}{b}+b_{2}\left(\frac{\delta}{b}\right)^{2}
$$

The parameters $a_{1}=1.0553, a_{2}=3.7997, b_{1}=3.8018$ and $b_{2}=2.7364$ were estimated by Maali over a wide range of Reynolds number $\left(R_{e}=10^{0}-10^{3}\right)$ by simply fitting the hydrodynamic expression of Sader to Eqs. 3 [23]. Parameter $\delta$, often called the boundary layer thickness, is the thickness of a thin viscous layer surrounding the cantilever over which the velocity has dropped by a factor of $1 / e(e=2.72)$, and can be given by [28]:

$$
\delta=\sqrt{\frac{\eta}{\pi \rho_{f} f}}
$$

where $\eta$ is the fluid's dynamic viscosity. Under the assumption that no interference exists between higher-order modes of vibration and the first-order mode, the transfer function $H(L, f)$ can be written as:

$$
H(L, f) \approx \frac{w(L, f)}{F_{e x c}(L, f)} \approx \frac{H_{0}}{1-\left(\frac{f}{f_{0}}\right)^{2}+2 \xi \frac{f}{f_{0}} j}
$$

This function is the ratio of the deflection at the free-end of the cantilever, $w(L, f)$, to the excitation force at the free-end $F_{e x c}(L, f)$. $H_{0}$ denotes the static value of the transfer function, $f_{0}$ and $\xi$ are, respectively, the eigenfrequency and the damping coefficient in the fluid which both depend on the terms $g_{1}(f)$ and $g_{2}(f)$, and $L$ is the cantilever length. This permits the imaginary and real parts of the normalized transfer function, with respect to the static value, to be obtained experimentally at each frequency, knowing the module $|H(f)|$ and the phase $\phi$. Consequently, $g_{1}(f)$ and $g_{2}(f)$ in Eqs. 2 can be calculated at each frequency by:

$$
g_{1}=-2 \pi m_{L}\left|\frac{H_{0}}{H}\right| \frac{f_{0, v a c}^{2}}{f} \sin \phi
$$




$$
g_{2}=m_{L}\left(\left(1-\left|\frac{H_{0}}{H}\right| \cos \phi\right)\left(\frac{f_{0, v a c}}{f}\right)^{2}-1\right)
$$

where $m_{L}(=m / L)$ is the mass per unit length of the cantilever, and $f_{0, v a c}$ is the resonant frequency of the cantilever in vacuum. Thus, the determination of the viscous and inertial parts of the drag force allows one to extract analytically at each frequency the viscosity and mass density of the fluid $[14,29]$. It is worth mentioning that this approach has been built on the assumption that the fluid has a negligible elasticity so that the elastic modulus $\left(G^{\prime}\right)$ tends to zero. This is not the case for complex fluids such as polymer and surfactant solutions where such fluids have a considerable variation of $G^{\prime}$ with the frequency.

The apparent viscosity $\eta$ in Eq. 4 should be replaced by the complex dynamic viscosity $\eta^{*}$ when investigating the frequency-dependent rheological properties of fluids. This complex viscosity is linked to the complex shear modulus $G^{*}$ by the equation

$$
\eta^{*}=\frac{G^{*}}{j \omega}=\eta^{\prime}-j \eta^{\prime \prime}=\frac{G^{\prime \prime}}{\omega}-j \frac{G^{\prime}}{\omega}
$$

where $\eta^{\prime}$ and $\eta^{\prime \prime}$ are, respectively, the real and imaginary components of the complex viscosity, and $\omega$ is the angular frequency $(\omega=2 \pi f)$. Substituting Eq. 7 in Eqs. 2 leads to the following expressions for the elastic $\left(G^{\prime}\right)$ and viscous $\left(G^{\prime \prime}\right)$ moduli:

$$
\begin{aligned}
G^{\prime} & =\frac{\left[D\left(g_{2} \omega-C\right)+B^{2}\right] Z-B g_{1}}{D^{2} Z} \\
G^{\prime \prime} & =\frac{g_{1}-B Z}{D}
\end{aligned}
$$

where $\quad D=\frac{\pi b_{2}}{2 \omega}, \quad B=\frac{\pi b b_{1} \sqrt{\rho_{f}}}{2 \sqrt{2}}, \quad C=\frac{\pi \rho_{f} b^{2}}{4} \omega \quad$ and $Z=\sqrt{\frac{-\left(B^{2}+2 D\left(g_{2} \omega-C\right)\right)+\sqrt{\left(B^{2}+2 D\left(g_{2} \omega-C\right)\right)^{2}+4 D^{2} g_{1}^{2}}}{2 D^{2}}}$ 
Preprint: Measurement Science and Technology, Vol. 23, 2012, 125306 (10pp)

In conclusion, $g_{1}(f)$ and $g_{2}(f)$ can be estimated from the amplitude and phase of deflection spectra of a cantilever immersed in a fluid, thereby permitting the viscoelastic rheological properties of the fluid $\left(G^{\prime}, G^{\prime \prime}, \eta^{*}\right)$ to be calculated from Eqs. 8 with a prior knowledge of the fluid's mass density.

\section{RESULTS AND DISCUSSION}

We illustrate the application of the proposed method to the study of the rheological behavior of several representative simple Newtonian (silicone oil) and complex fluids. We consider in particular two different types of complex fluids: surfactant WLM and polymer solutions. These solutions are characterized by their rich dynamics and unique mesoscopic structures that make them ideally suited for validating the proposed method. In addition, their high-frequency viscoelasticity has been studied recently using particular microrheological techniques such as DWS [30-34] and laser particle tracking [35-37]; thus, a detailed comparison between the proposed method and these techniques may be made.

\section{A. Linear viscoelasticity and thermal effect}

The frequency behavior of materials can give information on molecular structure at the quiescent state. Therefore, the applied force necessary to induce flow should be low enough to avoid destruction of the material's microstructure. Analogous to the strain sweep experiments in conventional rheology, actuation voltage sweep has been conducted to estimate the actuation voltage limit that will ensure operation in the linear viscoelastic regime. Figure 3 shows an example of the amplitude and phase of the ratio between the tip deflection of the LH cantilever immersed in the CPyCl1 solution and the actuation voltage at different voltage values. 


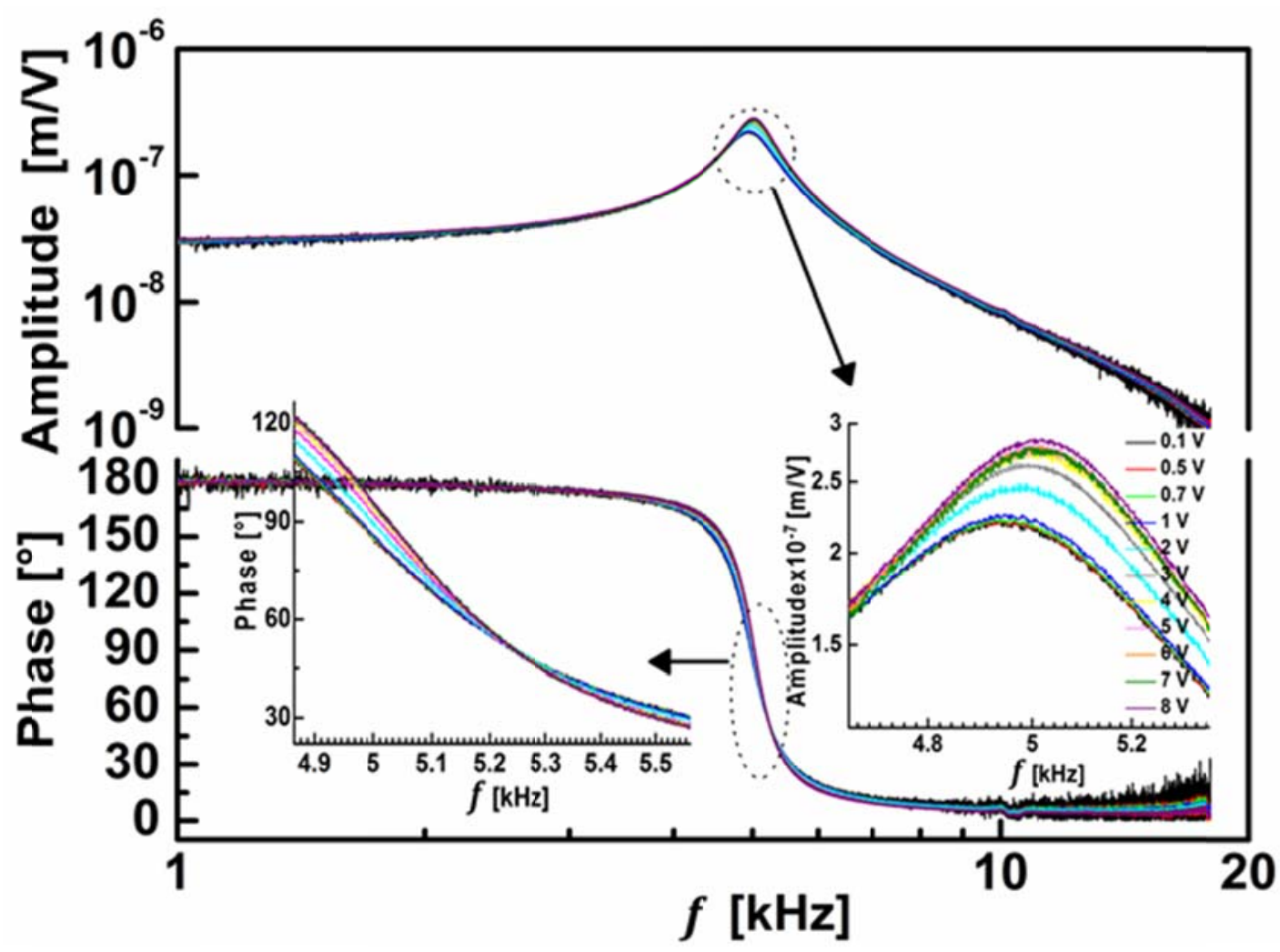

Figure 3. Spectrum of the cantilever deflection divided by the actuation voltage for LH cantilever immersed in CPyCl1 surfactant solution at different voltage values ranging from 0.1 to $8 \mathrm{~V}$. The insets represent the zoom view of amplitude and phase close to the resonant frequencies of the cantilever deflection divided by the actuation voltage.

It can be seen that raising the actuation voltage has no effect on the amplitude and phase spectra of deflection to voltage ratio until the $1 \mathrm{~V}$ level, beyond which the amplitude and resonant frequency $f_{r}$ differ. This can be better seen in figure 4(a), where the maximum normalized amplitudes $\left(H_{\max }\right)$ for both cantilevers $\mathrm{LH}$ and A remain almost constant until $1 \mathrm{~V}$, then increase as the voltage rises. Concurrently, the resonant frequency $f_{r}$ shows the same trend upon increasing the voltage. It is worth mentioning that the increase in $H_{\max }$ and $f_{r}$ with voltage increase of the in-fluid cantilevers is in contrast to the behavior of cantilevers operating in air, for which both $H_{\max }$ and $f_{r}$ decrease with an increase of the voltage as depicted in figure 4(b). Such a difference in behavior may be attributed to a thermal effects resulting from increasing the voltage in the two different environments. On the one hand, the Young's modulus of the cantilever in air decreases as the temperature increases [38], thus decreasing $f_{r}$. On the other hand, the heating effect reduces the viscosity of the $\mathrm{CPyCl} 1$ solution and the boundary layer thickness gradually decreases 
(according to Eq. 4) so that both $H_{\max }$ and $f_{r}$ increase with the voltage. The heating effect is more pronounced with the cantilever LH than with A as indicated by the difference between $H_{\max }$ values in both the up- and downscan modes (figure 4(a)). This might be the result of the relatively higher surface area of the LH cantilever in contact with the liquid. This physical interpretation of the thermal effect will be further investigated with numerical simulations.
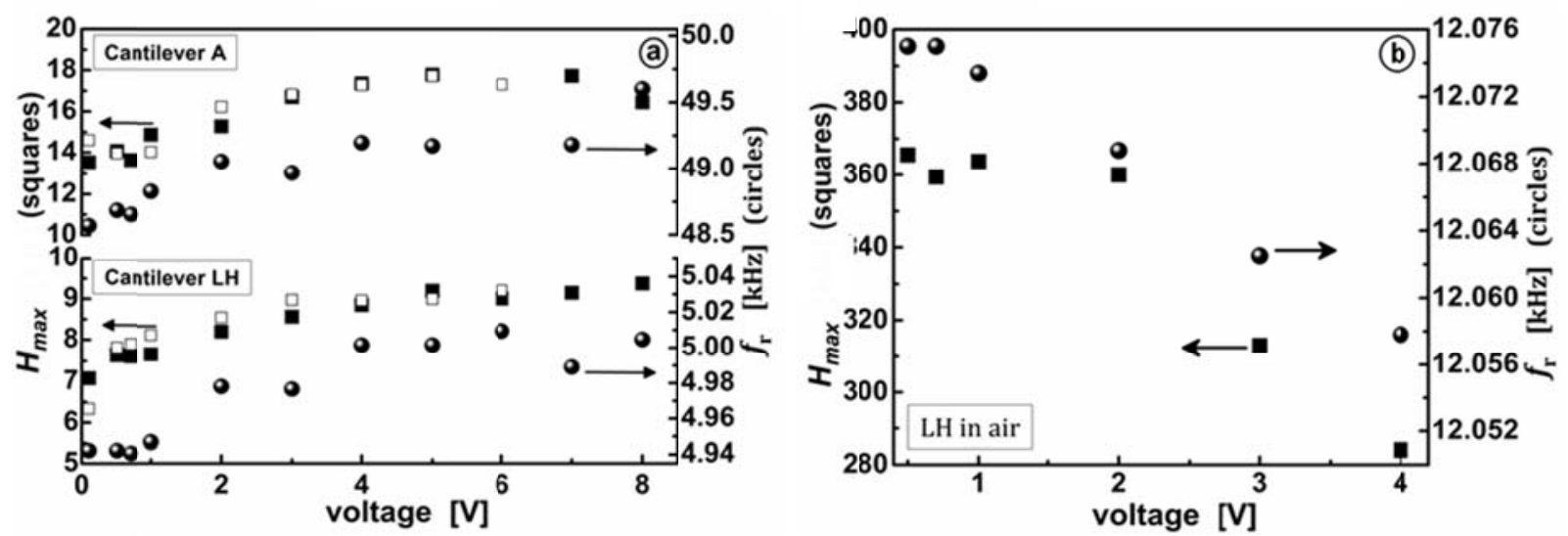

Figure 4. Variation of the maximum amplitude of deflection $\left(H_{\max }, \boldsymbol{)}\right)$ and the resonant frequency $\left(f_{r}\right.$, -) of the cantilever(s) (a) LH and A in CPyCl1 surfactant solution, and (b) LH in air. The open squares in figure 4(a) denote the downscan scenario. (The normalization of the amplitude is done for each cantilever with the amplitude measured for the smallest measurement frequency).

One volt was sufficient to induce the cantilever deflection with less noisy spectra and almost attain the thermal equilibrium, but the question remains: What is the effect on the fluid's microstructure and, thus, its linear viscoelasticity? For a vibrating cantilever in its out-of-plane mode, the shear stress amplitude $(\sigma)$ in the fluid can be simply calculating as follows:

$$
\sigma=\eta \dot{\gamma}(0, L, \omega)=-\frac{\omega \sqrt{\eta \rho_{f} \omega}}{\sqrt{2}} w(L, \omega)
$$

where $\dot{\gamma}$ is the shear rate, $\rho_{f}$ the fluid density and $\omega$ the angular frequency. (A detailed derivation of Eq. 9 is included in Supplementary Information.) Then, the complex shear modulus $\left|G^{*}\right|\left(=\sqrt{G^{\prime 2}+G^{\prime 2}}\right)$ can be estimated from Eqs. 8. Using the relation: $\gamma=\sigma / G^{*}$, the strain can be calculated. Note that the rheological parameters $\left(\sigma, \gamma, G^{*}\right)$ have been calculated at the resonant frequency, i.e., $\omega$ has been replaced by $2 \pi f_{r}$ in Eqs. 8, 9. 
Analogous to the strain sweep tests in conventional rheometers, voltage sweep tests can be conducted in our microrheological method and can successfully examine the linear viscoelastic regime. Figure 5 typically presents the variation of $G^{*}$ with the strain (at the resonant frequencies) for some examined complex fluids. Clearly evident in this figure is the fact that the shear modulus is almost independent of strain until a critical strain value is reached; beyond this level the shear modulus decreases, indicating a deviation from linear viscoelasticity. The critical strain value depends on the fluid microstructure and composition; e.g., it is 0.004 and 0.012 for $\mathrm{CTAB} 1$ and $\mathrm{CPyCl} 2$ surfactant solutions, respectively, and $4.3 \times 10^{-4}$ for the polymer solution. The corresponding critical voltage values can then be estimated from the inset of figure 5 , which demonstrates the linear increase of strain with the applied voltage for the LH cantilever, as an example. To ensure operation in the linear viscoelastic regime, $0.5 \mathrm{~V}$ is found to be the critical voltage used to actuate the LH cantilever immersed in PEO solution, whereas 0.8 and $1.6 \mathrm{~V}$ are the critical voltages in cases of CTAB1 and $\mathrm{CPyCl} 2$ solutions, respectively.

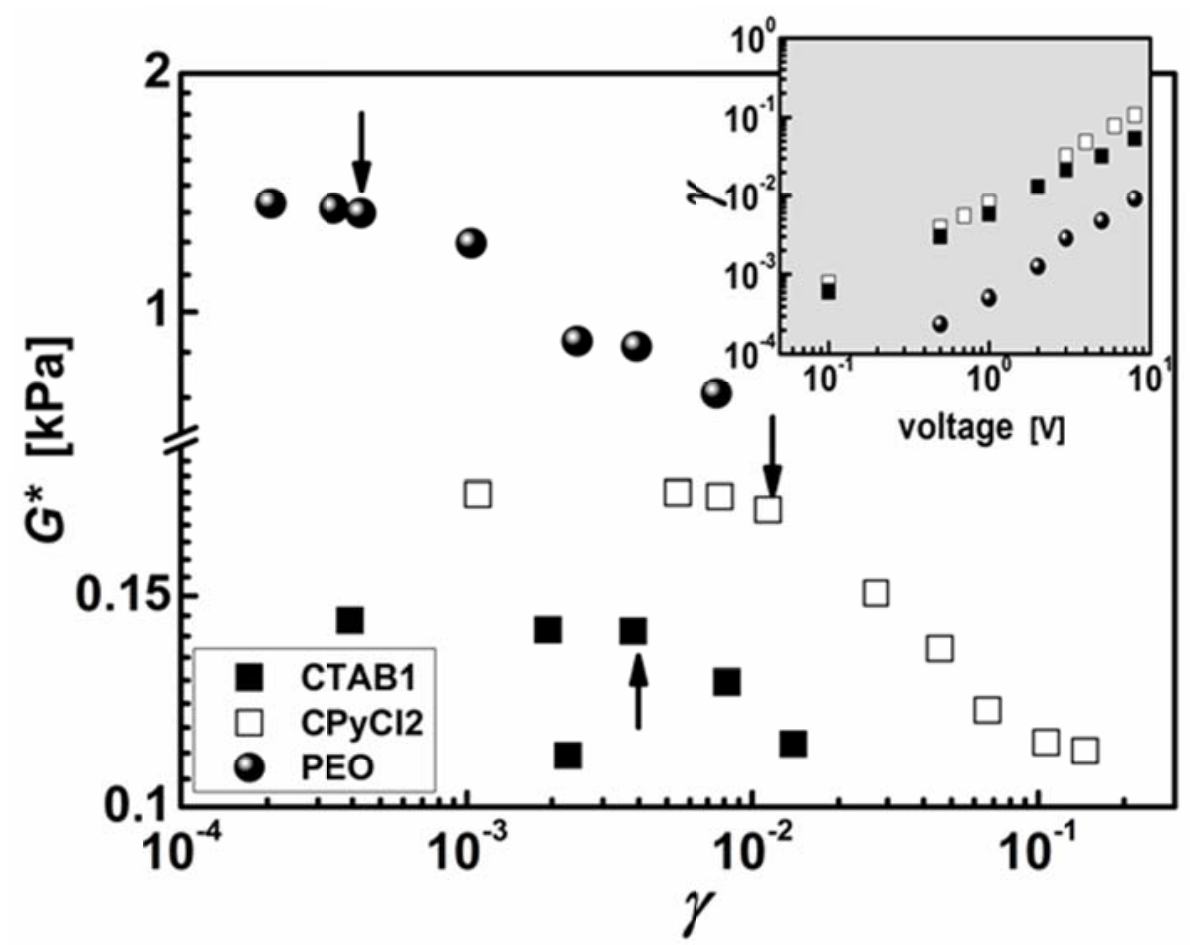

Figure 5. Strain sweep test: variation of the complex shear modulus $\left(G^{*}\right)$, at the resonant frequency, with strain $(\gamma)$ for different complex fluids using the cantilever $\mathrm{LH}$ at $20^{\circ} \mathrm{C}$. The inset shows the relation between the applied voltage and the corresponding strain at the resonant frequency of a cantilever LH immersed in complex fluids. The arrows indicate the critical strain for each fluid. 
For comparison, dynamic strain sweep tests have been conducted for the complex fluids at $1 \mathrm{~Hz}$. This is the common frequency at which such mechanical experiments are frequently done. In contrast to the microrheological data, the representative complex fluids show a decrease in the critical strain amplitudes as follow: PEO $>$ CTAB1 $\gtrsim C P y C l 2$. Such differences in both the trend and values of the critical strain may be attributed to the larger difference between the frequency at which the conventional rheology $(1 \mathrm{~Hz})$ and our microrheology $(\mathrm{kHz})$ were conducted. The slow dynamics and hence the bulk properties of the fluids are dominant at $1 \mathrm{~Hz}$; however, the microscopic properties of a single chain control the dynamic behavior at the $\mathrm{kHz}$ frequency scale.

\section{B. Microrheology of Newtonian fluid}

The first step in evaluating this method is to run a series of experiments on simple Newtonian liquids of known viscosity and mass density such as silicone oil. The highfrequency rheogram of 20 -mPa.s silicone oil, as an example, is depicted in figure 6. It is clear that the silicone oil behaves as a liquid-like Newtonian fluid since $G^{\prime \prime}$ is higher than $G^{\prime}$ and $\eta^{*}$ and is almost independent of the frequency. Generally, the overall trend of the dynamic moduli obtained by microrheology shows a quite good continuity with the macrorheology data, particularly with $G^{\prime \prime}$ which perfectly exhibits a scaling law $G^{\prime \prime} \sim \omega^{1}$ over six decades of frequency. The low-frequency $G^{\prime}$ of a Newtonian fluid usually tends to zero; therefore, a high degree of uncertainty in $G^{\prime}$ obtained by macrorheology is expected. 


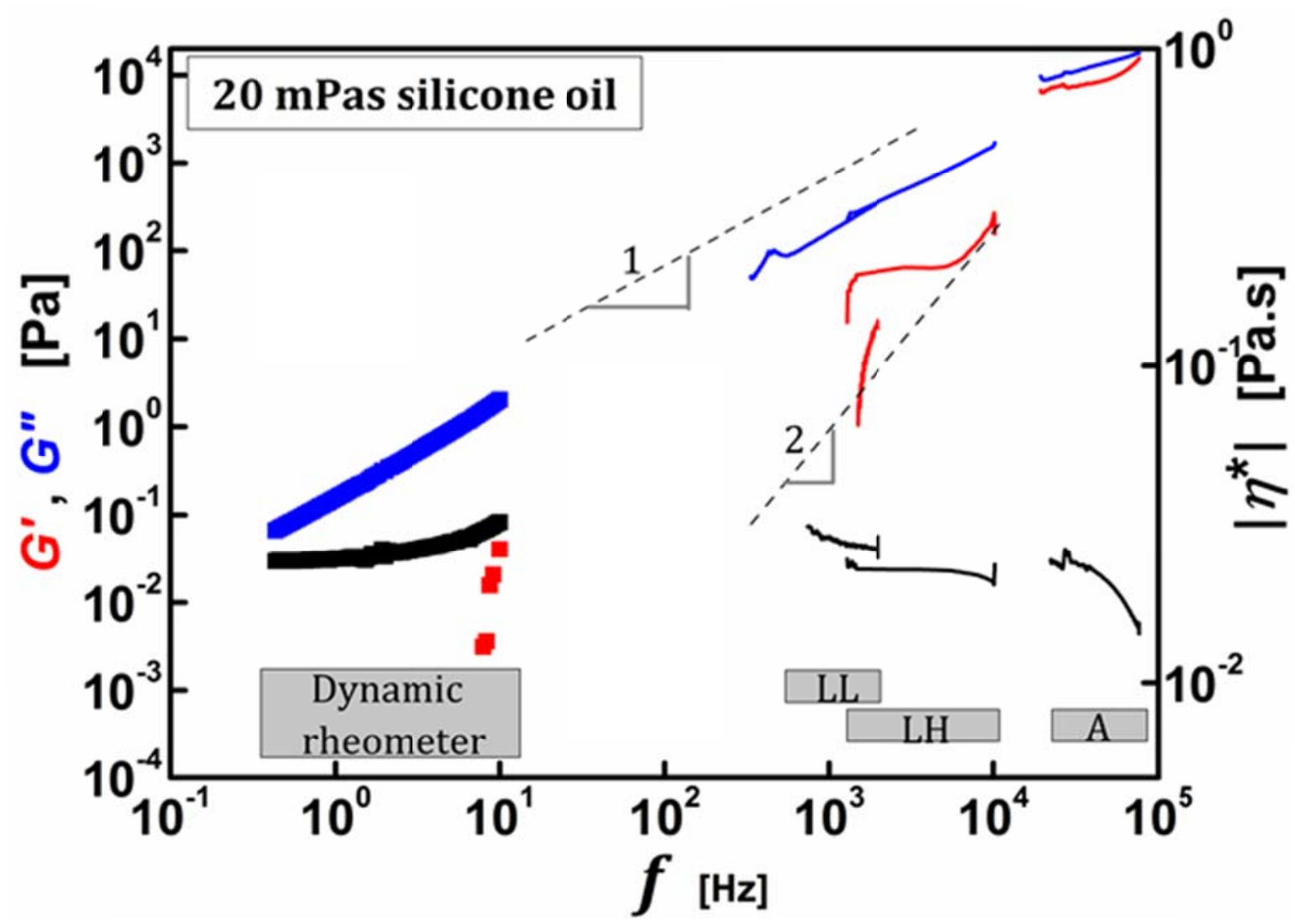

Figure 6. The elastic $G^{\prime}$ and viscous $G^{\prime \prime}$ moduli and complex viscosity $\eta^{*}$ for a 20-mPa.s silicone oil over an extended frequency range at $20{ }^{\circ} \mathrm{C}$. The symbols represent the macrorheology data whereas the lines represent the microrheology data using different cantilevers. The dashed lines represent the scaling laws related to the Maxwell tail $\left(G^{\prime \prime} \sim \omega^{1}, G^{\prime} \sim \omega^{2}\right)$.

Typically, $G^{\prime}$ shows a scaling law $G^{\prime} \sim \omega^{2}$ in the terminal flow regime which is almost the case of $G^{\prime}$ obtained by microrheology, particularly around the resonance peak. The deviations from this trend at the extremes of the $G^{\prime}$ curves (for both LL and LH; figure 6) may imply the restricted applicability of Eq. 8a over the frequency range of resonance peak and the sensitivity of the calculation of $G^{\prime}$ to the accuracy of mass density estimation. Accordingly, significant inconsistency between the elastic modulus patterns over the whole frequency range appears in figure 6. This issue will be discussed in a future analytical study. Nevertheless, a qualitative rheological description may be presented. Interestingly, the silicone oil behaves as a Newtonian fluid up to $c a .50 \mathrm{kHz}$, then a slight decrease in $\eta^{*}$ may imply a shear-thinning behavior (i.e., transition to non-Newtonian behavior) in tandem with a high-frequency crossover of the dynamic moduli at higher frequencies (ca. $100 \mathrm{kHz}$ ). 
Preprint: Measurement Science and Technology, Vol. 23, 2012, 125306 (10pp)

One of the greatest advantages of the proposed method is the large range of frequencies that it covers. This is immediately apparent from figure 6 which shows the high-frequency viscoelastic behavior of a Newtonian fluid that is not accessible by conventional rheometry because of its inertial constraint. Optics-based techniques such DWS $[1,39]$ and particle tracking $[2,35,36,40]$ aim to achieve the same goal in probing the high-frequency behavior of complex fluids. However, they have shown some drawbacks and limitations stemming from the significant contribution of the probe particle to the sample's viscoelasticity [39,41,42], possible particle-sample interactions [43-45], a limited medium ergodicity and opacity [46] and a specific mismatch between the medium and probe particles as well as special calibration and alignment issues related to the optical setup and time-consuming data treatment. In contrast, our approach relies on the absence of fluidcantilever chemical interactions since the cantilever's surface is isolated by a chemically inert inorganic layer. Also, no complicated setup and calibration issues are introduced, nor is data treatment required. Moreover, neither prior knowledge of the surface chemistry of the cantilever nor mismatches between the examined liquid and probe device are needed. Since the deflection spectra are collected via optical instrument (vibrometer), the opacity of fluids may be considered as a constraint; however, this limitation could be overcome by using electrical measurements (piezoresistance and gain-phase analyzer). However, a relatively turbid PEO solution and non-ergodic concentrated surfactant solutions could be examined using optical measurements as will be described below.

\section{Microrheology of polymer solution}

The first model complex fluid for testing our approach is the semidilute solution of polyethylene oxide (PEO) that forms a viscoelastic entanglement network. The macro- and microrheology of $5 \mathrm{wt}$ \% PEO solution is depicted in figure 7. It is evident from the plot that low- and high-frequency viscoelastic properties of the PEO solution are consistent and the combination of both techniques yields viscoelastic moduli over eight decades of frequency. In fact, the microrheology data are obtained by the cantilevers LL, LH and A, and the lack of a cantilever with longer geometry leads to the absence of dynamic moduli over the $10^{1}-10^{3}$ $\mathrm{Hz}$ range. However, the qualitative and, to some extent, the quantitative viscoelastic behaviors of the polymer solution are in good agreement with the results obtained by other 
groups for analogous polymer solutions using DWS [1,47] and optical tweezers [48]. Such a quantitative difference in the dynamic moduli is likely to be a consequence of different molecular weight, polymer polydispersity and even different reactive stocks. In general, the polymer solution behaves liquid-like at low frequency with solid-like behavior becoming more prominent as the frequency increases. The "speculative" low- and high-frequency crossovers of dynamic moduli (at $c a .10 \mathrm{~Hz}$ and $10 \mathrm{kHz}$, respectively) are in good accordance with that found for the same polymer solution [47]. The behavior of the complex viscosity perfectly complements the good overlap between the conventional rheology and the microrheology (figure 7). The polymer solution is Newtonian until $c a .10$ $\mathrm{Hz}$, and then a shear-thinning behavior is exhibited as the frequency increases as a result of chain alignments.

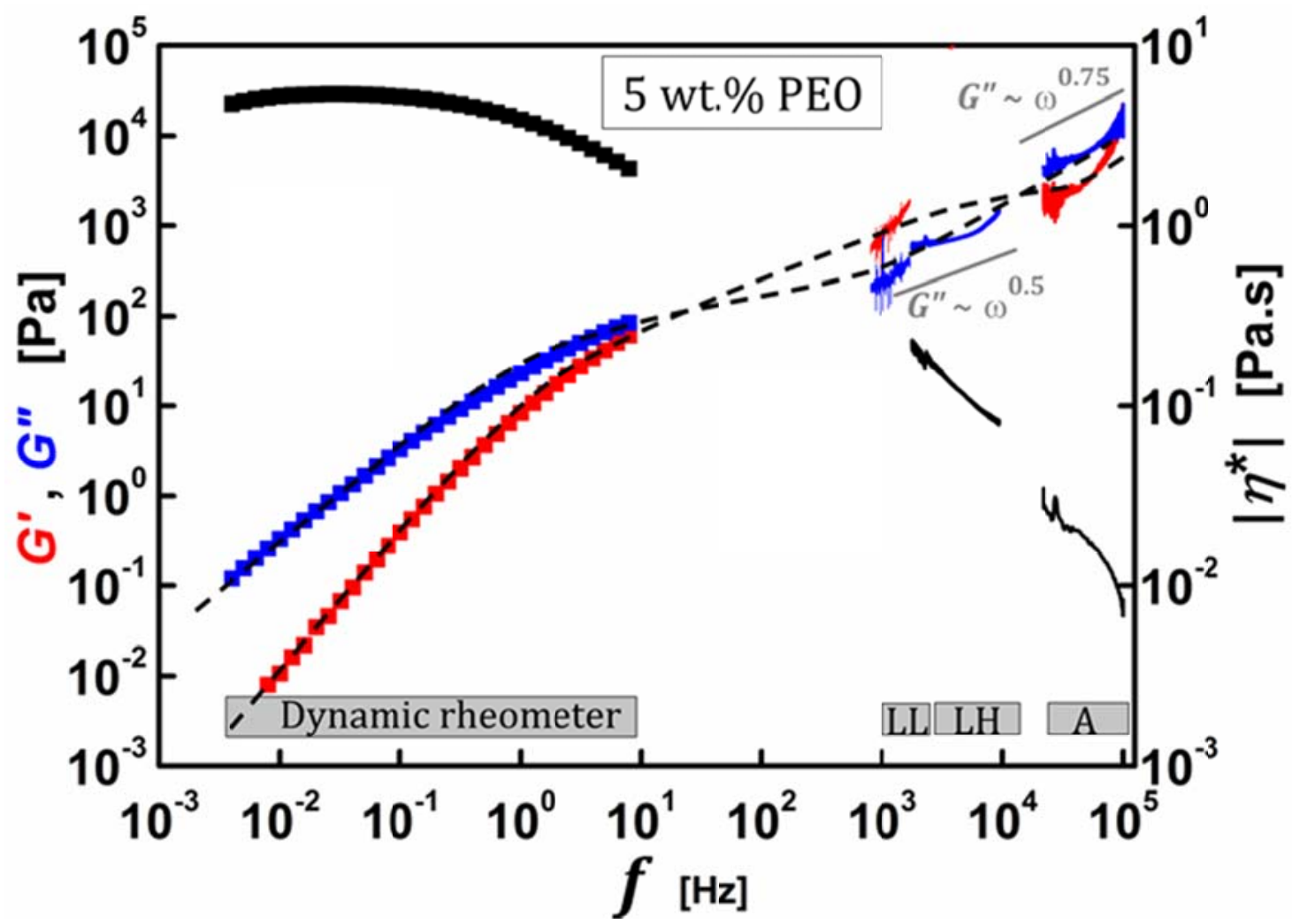

Figure 7. Viscoelastic spectra of an aqueous polymer solution of $5 \mathrm{wt} . \%$ PEO (400 kDa) at $20{ }^{\circ} \mathrm{C}$ obtained from macrorheology (symbols) and microrheology (lines). The dashed lines are visual guides, and the grey lines represent the high-frequency scaling laws.

The conventional rheometer data reveal the macroscopic (bulk) viscoelastic properties of the polymer network where the stress relaxation is governed by a reptation 
mechanism. Now, the importance of microrheology has been raised to precisely predict the viscoelastic behavior at higher frequencies, and also to deduce the stress relaxation mechanisms according to the scaling laws predicted by polymer theory. For flexible polymers, it is known that the shear modulus $G^{*}$ scales with the frequency, in the highfrequency limit, according to this relation: $G^{*} \sim \omega^{0.5-0.7}$ [49]. The exponent 0.5 implies the Rouse mode when no hydrodynamic effect exists and the flexible polymer chains can freely drain [50]. The uncertainty at the onset of the $G^{\prime}$ and $G^{\prime \prime}$ data obtained by our method, their varied slopes and the uncovered frequency ranges restrict making a precise statement about scaling exponents. However, we could carefully estimate a scaling exponent of 0.5 corresponding to the Rouse mode in agreement with previous findings for analogous flexible polymer solutions using particle tracking microrheology [40,51]. Beyond the highest crossover, a scaling exponent of 0.75 has been found in accordance with the predicted one by theoretical models, accounting for internal bending modes of Kuhn segments governing the stress relaxation mechanism at that frequency $[52,53]$.

\section{Microrheology of WLM solutions}

More complex models with very rich dynamic properties are the surfactant wormlike micellar solutions that drew considerable attention during the last decade to test modern microrheology techniques such as DWS [30-34] and particle tracking microrheology [3537]. Similarly, we examined our method with various WLM solutions. Quantitatively, the comparison of viscoelasticity of surfactant WLM systems from different authors is a challenge, since the measurement protocols, cell geometry, sample preparation, and even different reactive stocks have been described as factors that could introduce large deviations in the dynamic behavior of wormlike micelles. It is important to note that the estimation of the plateau modulus $G_{0}$ for different nominally identical samples have an error bar of 20\%; for relaxation times the error bar is 5\% [33]. As will be seen, microrheology also shares some of these problems. Therefore, this has to be considered when microrheology and mechanical rheology are compared in wormlike micelles. However, they cannot be considered the only origin of deviations in the viscoelastic data as will be described below. 
Preprint: Measurement Science and Technology, Vol. 23, 2012, 125306 (10pp)
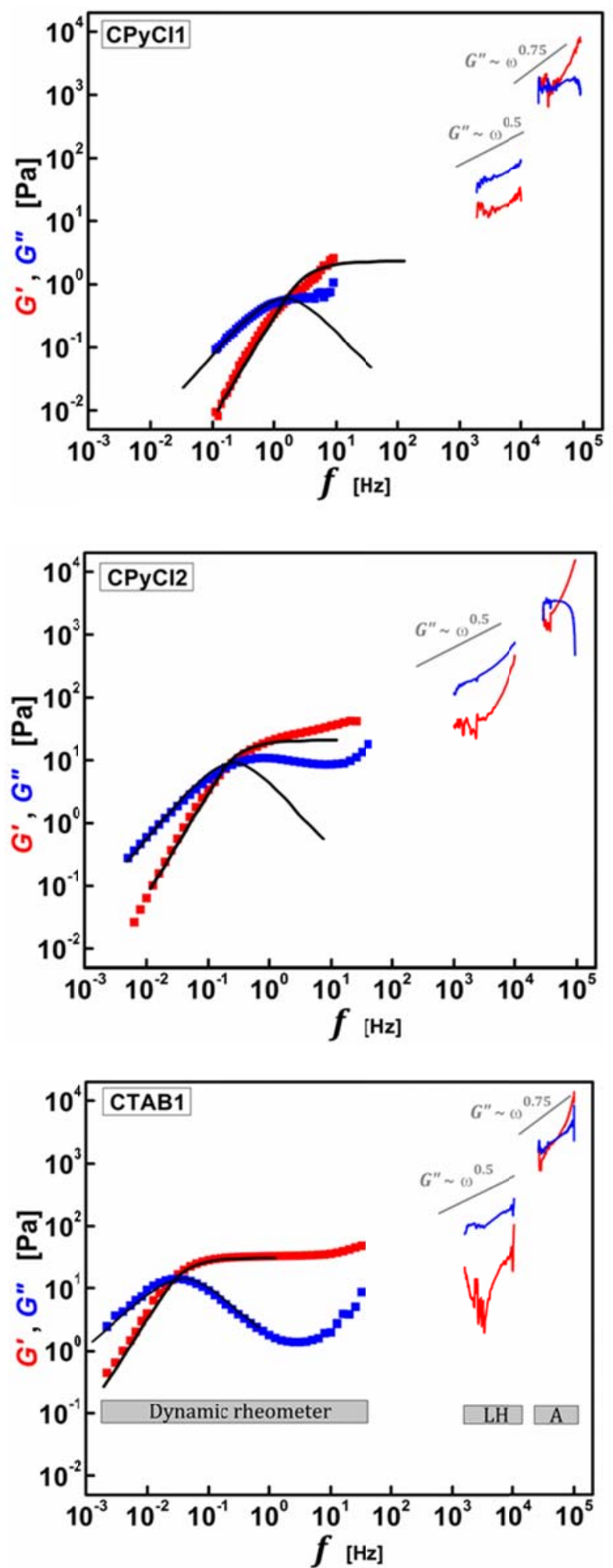

Figure 8. Viscoelastic spectra of various surfactant WLM solutions at $20{ }^{\circ} \mathrm{C}$ obtained from macrorheology (symbols), and microrheology (colored lines). The black lines are the best fit for a Maxwell model at low frequency, and the grey lines are the best fit for the high-frequency scaling laws. At constant NaSal-to- 
Preprint: Measurement Science and Technology, Vol. 23, 2012, 125306 (10pp)

surfactant molar ratios, the rheological behavior tends to fit the Maxwell model well as the surfactant concentration increases. This results from enough micellar growth to form a network that stress-relaxes via reptation and breaking-recombination mechanisms [54].

Figure 8 presents the high-frequency viscoelasticity extending beyond the Maxwell regime to cover eight decades of frequency. At high frequencies we expect an asymptotic crossover (corresponding to the shortest relaxation time; $\tau_{\min }$ ) from elastic response determined by collective network dynamics to response dominated by short-range single filament relaxation. In our experiments (figure 8), frequencies less than $1 \mathrm{kHz}$ are not accessible due to the lack of a cantilever that operates in this frequency range. Thus, the $G^{\prime}-$ $G^{\prime \prime}$ crossover at high frequency cannot be inferred since it typically appears in the frequency range beyond $1.6 \mathrm{kHz}$ for WLM solutions [31-33], far below the range of the cantilevers used here. However, the general viscoelastic behavior for the presented wormlike micelles is in a good agreement with the results presented previously for the same surfactant solutions $[33,35]$. Again, the uncertainty at the onset of the $G^{\prime}$ and $G^{\prime \prime}$ data (c.f. CTAB1 in figure 8) and the uncovered frequency ranges restrict making a precise statement about the scaling behavior of the dynamic moduli beyond $\tau_{\min }$. However, a scaling law $G^{\prime \prime} \sim \omega^{0.5}$ has been noticed in all examined WLM solutions (figure 8) indicating an intramicellar Rouse relaxation mode of the wormlike micelles, in accordance with a previously reported exponent [33] A closer inspection for the scaling relations of $G^{\prime \prime}$ at $f>50 \mathrm{kHz}$ (the crossover at the highest frequency) reveals that $G^{\prime \prime} \sim \omega^{0.75}$, reflecting the Kuhn segment bending mode in WLMs. The same scaling laws and thus the deduced relaxation mechanisms have been found in analogous WLM solutions using DWS [32-34]. It is worth noting that for WLMs the high-frequency shear moduli are essentially independent of temperature [32]; thus, the assumption that the heating effect induced by voltage application is not responsible for any uncertainty of moduli estimation.

In conclusion, our method provides high bandwidth frequency accessibility and much better defines the viscoelastic parameters than conventional rheometers, so that the microscopic properties of single chain and, thus, the associated relaxation mechanisms can be accurately estimated, in addition to the macroscopic (bulk) rheological properties, since the cantilever dimensions are much higher than the characteristic length scales (e.g. mesh 
size) of polymers or micellar networks. Another interesting advantage of our method is that it may be used to estimate the steady shear viscosity from the complex viscosity measurements. This can be done by applying the Cox-Merz rule through which information about nonlinear rheology at higher shear rates can be obtained from linear microrheology measurements. As can be seen from figure S(2), the polymer solution and diluted WLM solution of CPyCl1 perfectly obey Cox-Merz rule.

The calculation method used to relate the mechanical resonance response of an immersed microcantilever to the rheological properties of the medium requires an extremely precise estimation of the parameters involved in Eqs. 8 (resonant frequency in vacuum, static deflection and structural properties of the cantilever, density of the fluid). An error in one of these parameters strongly affects the calculated $G^{\prime}$ and $G^{\prime \prime}$. This point will be studied in detail in a future work. The static deflection of the cantilever (or static applied force) is difficult to measure independently with good accuracy. For instance, we need to measure the static deflection for the shortest cantilever (cantilever A) with a resolution less than one picometer and this measurement has to be done under identical conditions (temperature, type of fluid, electromagnetic applied force) as the resonant measurement. Results presented in this work are still affected by some error in this parameter estimation, especially the data far above or below the resonance frequency. A detailed analytical study covering this issue will appear in the near future.

\section{CONCLUSION AND PERSPECTIVES}

A microcantilever-based microrheometer has the potential to monitor the viscoelasticity of microliter-scale samples of Newtonian and complex fluids over a large frequency range ( 1 to $100 \mathrm{kHz}$ ). It has the capability to distinguish between the highfrequency dynamic behavior of various structured fluids, overcoming the limitations introduced by optical-based techniques (e.g. particle tracking and DWS) since it can measure viscoelastic moduli in the range of 0.01-10 kPa.

The approach presented herein can be integrated into arrays of microcantilevers on a unique chip to simultaneously measure the viscoelastic properties of soft matter and biological fluids in situ. Moreover, the non-linear rheology can be monitored as well if the 
Preprint: Measurement Science and Technology, Vol. 23, 2012, 125306 (10pp)

cantilever is placed in a microfluidic cell under pressure-driven flow. This will be the aim of future work.

\section{Acknowledgement}

The authors would like to thank Cédric Ayéla for the cantilevers mask design, and Liviu Nicu, Fabrice Mathieu and Daysuke Saya for the fabrication of the silicon cantilever. We thank Stephen Heinrich for his valuable comments on the manuscript. This work was partially supported by the CPER Pôle 4N Nanosciences en Aquitaine (GP-206-action 216/1) with the contribution of the Conseil Régional d'Aquitaine, the FEDER and the Ministry of Education and Research, by the French National Agency (MicRheo project $n^{\circ}$ ANR-08-NANO-004) and by the Conseil Régional d’Aquitaine (n²0091102001).

\section{References}

[1] Mason T G, Gang H and Weitz D A 1997 Diffusing-wave-spectroscopy measurements of viscoelasticity of complex fluids J. Opt. Soc. Am. A 14 139-149

[2] Mason T G, Ganesan K, van Zanten J H, Wirtz D and Kuo S C 1997 Particle tracking microrheology of complex fluids Phys. Rev. Lett. 79 3282-3285

[3] Kovacs G T A 1998 Micromachined Transducers (New York: McGraw-Hill Companies)

[4] Fadel L, Lochon F, Dufour I and Français 02004 Chemical sensing: millimeter size resonant microcantilever performance J. Micromech. Microeng. 14 S23-S30

[5] Vidic A, Then D and Ziegler Ch 2003 A new cantilever system for gas and liquid sensing Ultramicroscopy 97 407-416

[6] Lavrik N V, Sepaniak M J and Datskos P G 2004 Cantilever transducers as a platform for chemical and biological sensors Rev. Sci. Instrum. 75 2229-2254

[7] Waggoner Ph S and Craighead H G 2007 Micro- and nanomechanical sensors for environmental, chemical, and biological detection Lab Chip 7 1238-1255

[8] Zougagh M and Ríos A 2009 Micro-electromechanical sensors in the analytical field Analyst 134 1274-1290

[9] Wang Y-H, Chen C-P, Chang C-M, Lin C-P, Lin C-H, Fu L-M and Lee C-Y 2009 MEMSbased gas flow sensors Microfluid. Nanofluid. 6 333-346

[10] Dufour I, Josse F, Heinrich S M, Lucat C, Ayela C, Ménil F and Brand 02012 Unconventional uses of microcantilevers as chemical sensors in gas and liquid media Sens. Actuators, $B$ in press 
Preprint: Measurement Science and Technology, Vol. 23, 2012, 125306 (10pp)

[11] Jakoby B, Ecker A and Vellekoop M J 2004 Monitoring macro- and microemulsions using physical chemosensors Sens. Actuators, A 115 209-214

[12] Agoston A, Keplinger F and Jakoby B 2005 Evaluation of a vibrating micromachined cantilever sensor for measuring the viscosity of complex organic liquids Sens. Actuators, $A$ 123-124 82-86

[13] Zhao Y, Li S, Davidson A, Yang B, Wang Q and LinQ 2007 A MEMS viscometric sensor for continuous glucose monitoring J. Micromech. Microeng. 17 2528-2537

[14] Belmiloud N, Dufour I, Colin A and Nicu L 2008 Rheological behavior probed by vibrating microcantilevers Appl. Phys. Lett. 92 041907-10

[15] Smith P D, Young R C D and Chatwin C R 2010 A MEMS viscometer for unadulterated human blood Measurement 43 144-151

[16] Christopher G F, Yoo J M, Dagalakis N, Hudson S D and Migler K B 2010 Development of a MEMS based dynamic rheometer Lab Chip 10 2749-2757

[17] Rotsch C, Jacobson K and Radmacher M 1999 Dimensional and mechanical dynamics of active and stable edges in motile fibroblasts investigated by using atomic force microscopy PNAS 96 921-926

[18] Mahaffy R E, Shih C K, MacKintosh F C and Käs J 2000 Scanning Probe-Based Frequency-Dependent Microrheology of Polymer Gels and Biological Cells Phys. Rev. Lett. 85 880-883

[19] Du B, Tsui O K C, Zhang Q and He T 2001 Study of Elastic Modulus and Yield Strength of Polymer Thin Films Using Atomic Force Microscopy Langmuir 17 3286-3291

[20] Kawakami M, Byrne K, Khatri B S, McLeish T C B and Smith D A 2006 Viscoelastic Properties of Single Poly(ethylene glycol) Molecules Chem. Phys. Chem. 7 1710-1716

[21] Okajima T, Tanaka M, Tsukiyama S, Kadowaki T, Yamamoto S, Shimomura M and Tokumoto H 2007 Stress relaxation of HepG2 cells measured by atomic force microscopy Nanotechnology 18 084010-15

[22] Ogawa T, Kurachi S, Kageshima M, Naitoh Y, Li Y J and Sugawara Y 2010 Step response measurement of AFM cantilever for analysis of frequency-resolved viscoelasticity Ultramicroscopy 110 612-617

[23] Maali A, Hurth C, Boisgard R, Jai C, Cohen-Bouhacina T and Aimé J-P 2005 Hydrodynamics of oscillating atomic force microscopy cantilevers in viscous fluids J. Appl. Phys. 97 074907-12

[24] Youssry M, Belmiloud N, Caillard B, Ayela C, Pellet C and Dufour I 2011 A straightforward determination of fluid viscosity and density using microcantilevers: From experimental data to analytical expressions Sens. Actuators, A 172 40-46 
Preprint: Measurement Science and Technology, Vol. 23, 2012, 125306 (10pp)

[25] Blom F R, Bouwstra S, Elwenspoek M and Fluitman J H J 1992 Dependence of the quality factor of micromachined silicon beam resonators on pressure and geometry J. Vac. Sci. Technol., B 10 19-26

[26] Dufour I, Heinrich S and Josse F 2007 Theoretical Analysis of Strong-Axis Bending Mode Vibrations for Resonant Microcantilever (Bio) Chemical Sensors in Gas or Liquid Phase J. Microelectromech. Syst. 16 44-49

[27] Sader J E 1998 Frequency response of cantilever beams immersed in viscous fluids with applications to the atomic force microscope J. Appl. Phys. 84 64-76

[28] Batchelor G K 1974 Fluid Dynamics (Cambridge: Cambridge University Press)

[29] Belmiloud N, Dufour I, Nicu L, Colin A and Pistré J 2006 Vibrating Microcantilever used as Viscometer and Microrheometer IEEE Sensors Conference 753-756

[30] Bellour M, Skouri M, Munch J-P and Hébraud P 2002 Brownian motion of particles embedded in a solution of giant micelles Eur. Phys. J. E 8 431-436

[31] Cardinaux F, Cipelletti L, Scheffold F and Schurtenberger P 2002 Microrheology of giant-micelle solutions Europhys. Lett. 57 738-744

[32] Willenbacher N, Oelschlaeger C, Schopferer M, Fischer P, Cardinaux F and Scheffold F 2007 Broad Bandwidth Optical and Mechanical Rheometry of Wormlike Micelle Solutions Phys. Rev. Lett. 99 068302-05

[33] Galvan-Miyoshi J, Delgado J and Castillo R 2008 Diffusing wave spectroscopy in Maxwellian fluids Eur. Phys. J. E 26 369-377

[34] Sarmiento-Gomez E, Lopez-Diaz D and Castillo R 2010 Microrheology and characteristic lengths in wormlike micelles made of a zwitterionic surfactant and SDS in brine J. Phys. Chem. B 114 12193-12202

[35] Buchanan M, Atakhorrami M, Palierne J F, MacKintosh F C and Schmidt C F 2005 Highfrequency microrheology of wormlike micelles Phys. Rev. E 72 011504-09

[36] Buchanan M, Atakhorrami M, Palierne J F and Schmidt C F 2005 Comparing macrorheology and one- and two-point microrheology in wormlike micelle solutions Macromolecules 38 8840-8844

[37] Atakhorrami M and Schmidt C F 2006 High-bandwidth one- and two-particle microrheology in solutions of wormlike micelles Rheol. Acta 45 449-456

[38] Mertens J, Finot E, Thundat T, Fabre A, Nadal M-H, Eyraud V and Bourillot E 2003 Effects of temperature and pressure on microcantilever resonance response Ultramicroscopy 97 119-126

[39] Scheffold F, Skipetrov S E, Romer S and Schurtenberger P 2001 Diffusing-wave spectroscopy of nonergodic media Phys. Rev. E 63 61404-14 
Preprint: Measurement Science and Technology, Vol. 23, 2012, 125306 (10pp)

[40] Gittes F, Schnurr B, Olmsted P D, MacKintosh F C and Schmidt C F 1997 Microscopic viscoelasticity: Shear moduli of soft materials determined from thermal fluctuations Phys. Rev. Lett. 79 3286-3289

[41] Krall A H and Weitz D A 1998 Internal Dynamics and Elasticity of Fractal Colloidal Gels Phys. Rev. Lett. 80 778-781

[42] Romer S, Scheffold F and Schurtenberger P 2000 Sol-Gel Transition of Concentrated Colloidal Suspensions Phys. Rev. Lett. 85 4980-4983

[43] Verma R, Crocker J C, Lubensky T C and Yodh A G 2000 Attractions between Hard Colloidal Spheres in Semiflexible Polymer Solutions Macromolecules 33 177-186

[44] Starrs L and Bartlett P 2003 One- and two-point micro-rheology of viscoelastic media J. Phys.: Condens. Matter 15 S251-S256

[45] Mizuno D, Head D A, MacKintosh F C and Schmidt C F 2008 Active and Passive Microrheology in Equilibrium and Nonequilibrium Systems Macromolecules 41 7194-7202

[46] Breedveld V and Pine D J 2003 Microrheology as a tool for high-throughput screening J. Mater. Sci. 38 4461-4470

[47] Dasgupta B R, Tee S-Y, Crocker J C, Frisken B J and Weitz D A 2002 Microrheology of polyethylene oxide using diffusing wave spectroscopy and single scattering Phys. Rev. E 65 051505-14

[48] Tassieri M, Gibson G M, Evans R M L, Yao A M, Warren R, Padgett M J and Cooper J M 2010 Measuring storage and loss moduli using optical tweezers: Broadband microrheology Phys. Rev. E 81 026308-12

[49] Doi M and Edwards S F 1986 The Theory of Polymer Dynamics (UK: Oxford University Press)

[50] Rouse P E 1953 A Theory of the Linear Viscoelastic Properties of Dilute Solutions of Coiling Polymers J. Chem. Phys. 21 1272-1280

[51] Schnurr B, Gittes F, MacKintosh F C and Schmidt C F 1997 Determining Microscopic Viscoelasticity in Flexible and Semiflexible Polymer Networks from Thermal Fluctuations Macromolecules 30 7781-7792

[52] Gittes F and MacKintosh F C 1998 Dynamic shear modulus of a semiflexible polymer network Phys. Rev. E 58 R1241-R1244

[53] Morse D C 1998 Viscoelasticity of tightly entangled solutions of semiflexible polymers Phys. Rev. E 58 R1237-R1240 
Preprint: Measurement Science and Technology, Vol. 23, 2012, 125306 (10pp)

[54] Cates M E 1987 Reptation of living polymers: dynamics of entangled polymers in the presence of reversible chain-scission reactions Macromolecules 20 2289-2296; 1988 Dynamics of living polymers and flexible surfactant micelles: scaling laws for dilution $J$. Phys. II 49 1593-1600; 1996 Flow behaviour of entangled surfactant micelles J. Phys.: Condens. Matter 8 9167-9176; Cates M E and Candau S J 1990 Statics and dynamics of worm-like surfactant micelles J. Phys. Condens. Matter 2 6869-6892. 